\title{
Reference Breast Phantoms for Low-Cost Microwave Imaging
}

\author{
Emine Avşar Aydin*, Selin Yabaci Karaoğlan
}

\begin{abstract}
Microwave imaging provides an alternative method for breast cancer screening and the diagnosis of cerebrovascular accidents. Before a surgical operation, the performance of microwave imaging systems should be evaluated on anatomically detailed anthropomorphic phantoms. This paper puts forward the advances in the development of breast phantoms based on 3D printing structures filled with liquid solutions that mimic biological tissues in terms of complex permittivity in a wide microwave frequency band. In this paper; four different experimental scenarios were created, and measurements were performed, and although there are many vector network analyzers on the market, the miniVNA used in this study has been shown to have potential in many biomedical applications such as portable computer-based breast cancer detection studies. We especially investigated the reproducibility of a particular mixture and the ability of some mixes to mimic various breast tissues. Afterwards, the images similar to the experimentally created scenarios were obtained by implementing the inverse radon transform to the obtained data.
\end{abstract}

Keywords: cancer; conductivity; microwave imaging; permittivity; phantoms

\section{INTRODUCTION}

Breast cancer ranks second among female deaths all over the world. Breast cancer incidence is increasing in the United States and European Union countries [1].

Mammography facilitates the treatment of cancer by non-invasive techniques for the detection of small breast lesions $[2,3]$. Recently, more new treatments as opposed to traditional surgery have been investigated to meet these demands. Microwave imaging technology for breast cancer detection has attracted much attention. The physical basis for the detection of breast cancer with microwave imaging is the difference between the dielectric properties of normal and malignant breast tissues. The significant difference between the dielectric properties of the normal and malignant breast tissue in microwave frequencies, the number of misseddetections and, false-positives are meagre, and this is considered to be the most important motivation for developing a microwave imaging technique for breast cancer detection. The contrast between the estimated malign-tonormal breast tissue is between 2:1 and 10:1, depending on the healthy tissue density. Another advantage of the microwave imaging technique is that the respective tissue properties are shown in a three-dimensional (3-D) volumetric map. Furthermore, microwave imaging is a non-invasive and even potentially a low-cost alternative to breast cancer imaging. For all the reasons mentioned above, microwave breast imaging has the potential to overcome some of the limitations of traditional breast cancer screening systems.

One of the most critical factors in the effective treatment of breast cancer is early diagnosis [4-6]. Early diagnosis is crucial both in increasing the success rate of treatment and decreasing the treatment costs. Hence, breast imaging is essential for early diagnosis and effective treatment.

There are many different techniques for breast imaging: mammography, ultrasound and magnetic resonance imaging [4-10]. The most common method used in breast imaging is mammography imaging, which provides a 2D image of the compressed breast. However, mammography may be inadequate in early and accurate diagnosis due to the constraints caused by the overlapping of the displayed tissues.

Numerical breast microwave imaging is a new and increasingly widespread imaging technique that can display a high-resolution 3D imaging of the breast by using a limited number of 2D scans obtained with a limited scanning angle. This feature can be displayed in slices of the breast image obtained from 3D and it allows for a more detailed examination with in-depth information.

Numerical breast microwave imaging reduces the problems of traditional mammography imaging, such as the over-tightening of the breast during imaging and the overlapping of tissues, and the inability to overlook abnormal structures in the breast, whereas more dose problems arise [13].

The amount of the dose should be reduced so that it is an $\mathrm{X}$-ray based imaging and that the X-ray can be used as a routine screening method due to the ionizing radiation effect. For this purpose, two-way studies have been carried out, including studies on hardware improvements and studies on image retraction techniques.

It is possible to obtain low dose images with developing a detector technology [1-15]. On the other hand, dose reduction is possible with compressed detection-based backscattering techniques using a small number of projections. The use of the digital breast microwave imaging technique in breast imaging will increase in proportion to the decreasing dose amount.

Imaging techniques should have high sensitivity and sensitivity. However, the ease of use, low interference, high spatial resolution for sharp interpretation, low cost, etc. are sought after.

In recent years, an approach has been introduced that allows the imaging of the breast with a non-linear regression algorithm using a microwave-based imaging and electromagnetic radiation at a frequency of around $1 \mathrm{GHz}$ [11-14].

Microwave applications [1-15] have been used to determine the dielectric properties of the living tissues such as fat, muscle, etc. These waves penetrating the human body 
are scattered depending on the electrical properties of the displayed tissues. It is possible to map the electrical properties of the displayed tissues by processing the scattered waves by using a suitable model.

Electrical permittivity and conductivity are two critical parameters that determine the diffusion of microwaves in materials [15]. Different biological tissues in the microwave frequency range cause different spreading behavior. For example, fat and skin have low permittivity values, while blood and cancerous tissues have higher values. If the responses of the target tissues to the microwave signals are processed together in different angles, they allow the internal structure of the tissues to be visualized.

The back-scattering operation in Microwave Imaging is the process of solving the inverse problem by using the data collected by the antenna and scattered from the target. A cost function is defined to perform the back-scattering operation. The basic idea in the back-scattering algorithm is to update the displayed dielectric properties of the displayed target to minimize the difference of the calculated model.

Since microwave breast imaging is not an ionizing radiation hazard, there is a chance of being an efficient and reliable cancer detection method. In this study, four different experimental scenarios were created, and measurements were taken. Although there are many vector network analyzers on the market, miniVNA used in this study has been found to have a great potential in many biomedical applications, such as breast cancer detection studies, because of being more affordable, portable and computer-based.

However, many experimental systems and imaging algorithms have been studied, and promising results have already been obtained, using both radar-based and inverse scattering reconstruction algorithms. On the other hand, before inversion algorithms can be used in clinical studies, they need to be tested against the experimental data collected in controlled configurations on reference phantoms. Although the latter must be close to breast tissues in terms of dielectric properties, simple structures benefit instability over time and reproducibility over more complex structures.

\section{HOMOGENOUS PHANTOMS PRODUCTION}

To imitate a real human breast and tumor tissues, four different mixtures were produced in the form of "water-oil in gelatin" dispersions. Phantoms were built to simulate the real breast skin, fat, gland and tumor tissues in their dielectric properties. This method was chosen because of the simple style of the fabrication and the ease when obtaining the materials, stable mechanical properties and a long storage time $[16,17]$. As for the chemicals, p-toluic acid, n-propanol and formaldehyde were purchased from Merck (Darmstadt, Germany). For deionized water, Elga Purelab Option-Q (UK) was used. 200 bloom gelatin (Tito, Turkey) and sunflower oil (Komili, Turkey) were purchased from the local market. Persil liquid detergent was used (Henkel, Germany) for the analysis. The specific amounts of the chemicals used in the phantom fabrication can be seen in Tab. 1. The method was adapted from Lazebnik, Madsen [18]. The production method of the phantom is summarized as a flow diagram in Fig. 1.

Table 1 The specific amounts of the chemicals used in the phantom's fabrication

\begin{tabular}{|l|c|c|c|c|}
\hline \multirow{2}{*}{\multicolumn{1}{c|}{ Material }} & \multicolumn{4}{c|}{ Quantity } \\
\cline { 2 - 5 } & Fat & Gland & Skin & Tumor \\
\hline p-toluic acid $(\mathrm{g})$ & 0.133 & 0.253 & 0.294 & 0.346 \\
\hline n-propanol $(\mathrm{mL})$ & 6.96 & 12.71 & 28.69 & 17 \\
\hline deionized water $(\mathrm{mL})$ & 132.7 & 241.9 & 279.5 & 328 \\
\hline 200 Bloom gelatin $(\mathrm{g})$ & 24.32 & 43.27 & 50.02 & 58.67 \\
\hline Formaldehyde * $(\mathrm{g})$ & 1.53 & 2.74 & 3.33 & 3.72 \\
\hline oil $(\mathrm{mL})$ & 269.6 & 141.5 & 98.6 & 38.4 \\
\hline Detergent $(\mathrm{mL})$ & 12 & 6.79 & 5.86 & 2 \\
\hline *37\% by weight & & & &
\end{tabular}

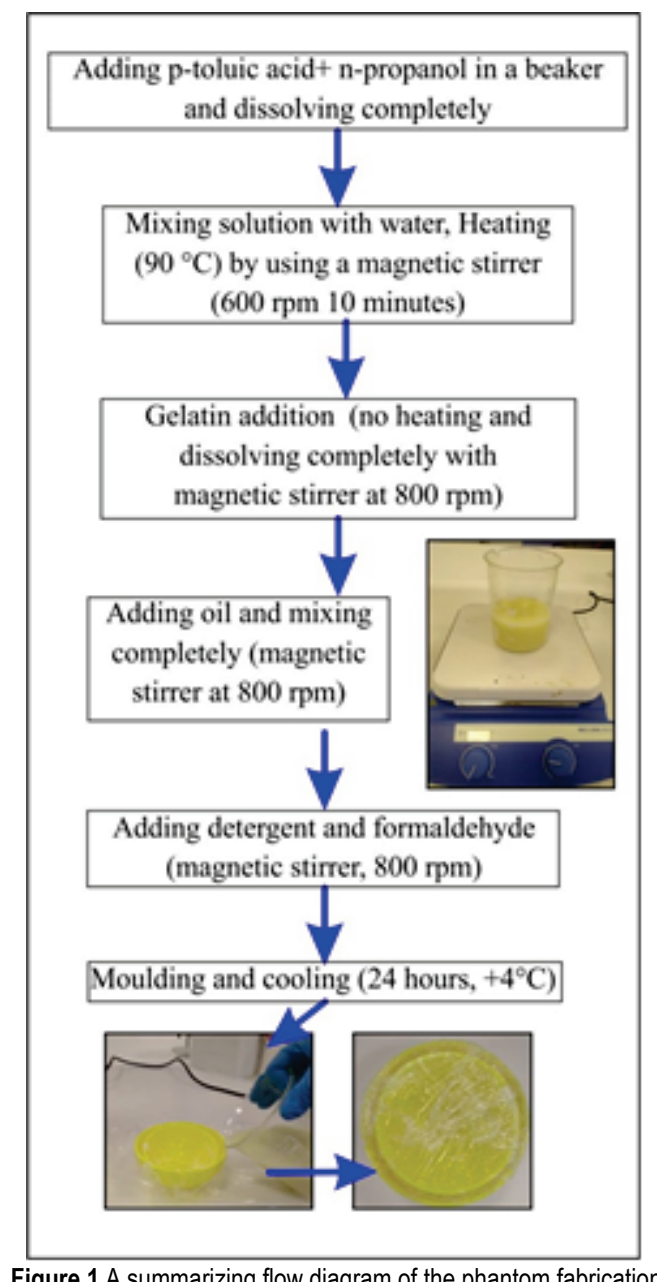

Firstly, p-toluic acid and n-propanol dissolved completely. The water part was then added with heating and stirring (by a magnetic stirrer- $90{ }^{\circ} \mathrm{C}, 600 \mathrm{rpm}$ ). After 10 minutes, the heating was stopped, and gelatin was added to the mixture to create a gel matrix with continuous stirring (800 rpm). After gelatin dissolved completely, the oil part was added into the matrix system, again with continuous stirring $(800 \mathrm{rpm})$. At the end, detergent was added to emulsify the water and oil part and formaldehyde was added to preserve the system, homogenously. The mixture ("wateroil in gelatin" system) was molded and cooled in a refrigerator $\left(+4{ }^{\circ} \mathrm{C}, 24\right.$ hours) to trap water and oil in the 
gelatin matrix system and to solidify. Fig. 2 shows the last shape of the molded phantom. Tumor production was done in four different sizes such as 0.5, 1.0, 1.5 and $2 \mathrm{~cm}$ spherically, and it can be seen in Figure 3. For the analysis, these tumors were placed both in the center and sides of the breast phantom. Tissues should properly be stored without any air or moisture exposure. This situation can change the dielectric properties of the system. When they are closed hermetically by a plastic wrap or a container, the tissues will retain their structure for a longer time.

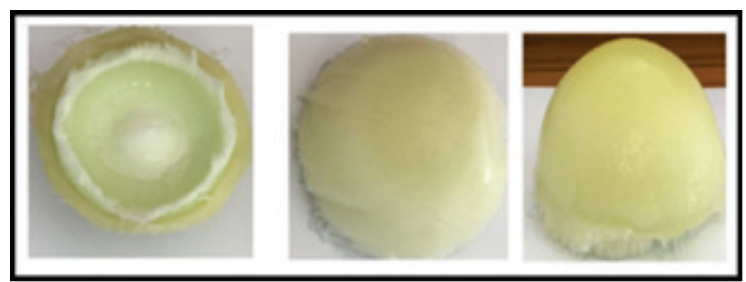

Figure 2 Breast phantom layers and the whole state

\section{BREAST PHANTOMS' MEASUREMENT}

The VNA contains two ports: one is used to send the signal, whereas the other receives the signal simultaneously. As shown in Fig. 3, two types of measurements are made by the VNA system: the forward and backward measurement [19].

In forward measurement, the signals are separated from port 1 , and the signals are separated from port 2 in the inverse measurement [19]. If signals are emitted from a port and reflected back to the same port, this is called the reflection measurement. Otherwise, if the signal is output from a port and forwarded to the other port, it is considered as a transmission measurement.

S-parameters provide complete information about the linear behavior of RF and microwave components and are independent of the properties of the VNA [19]. By courtesy of VNA calibration, the device's defects are entirely removed from the measurement. Thereupon, the S-parameters are a highly delicate presentation of how the linear behavior of the tested component describes how it behaves and how it interacts with other devices when cascaded.

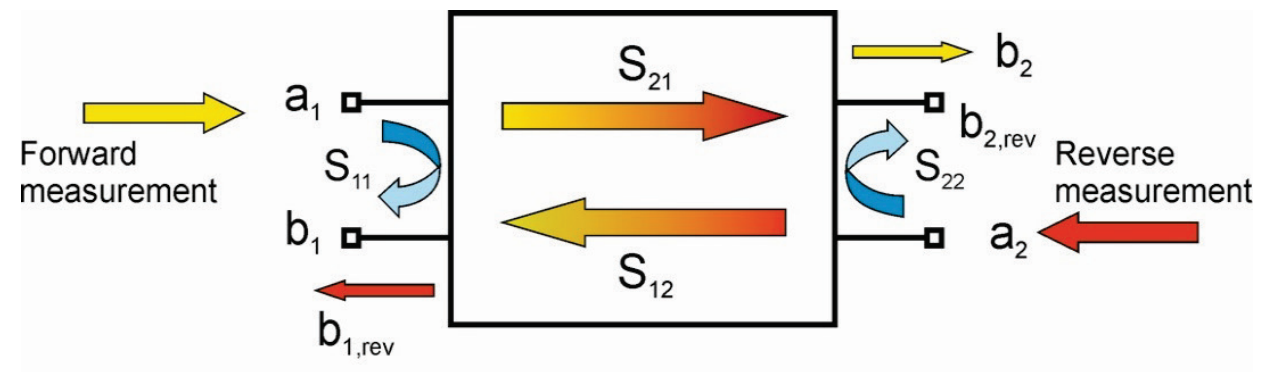

\section{Port 1}

\section{DUT}

Figure 3 Scattering parameters of a two-port network

\section{Port 2}

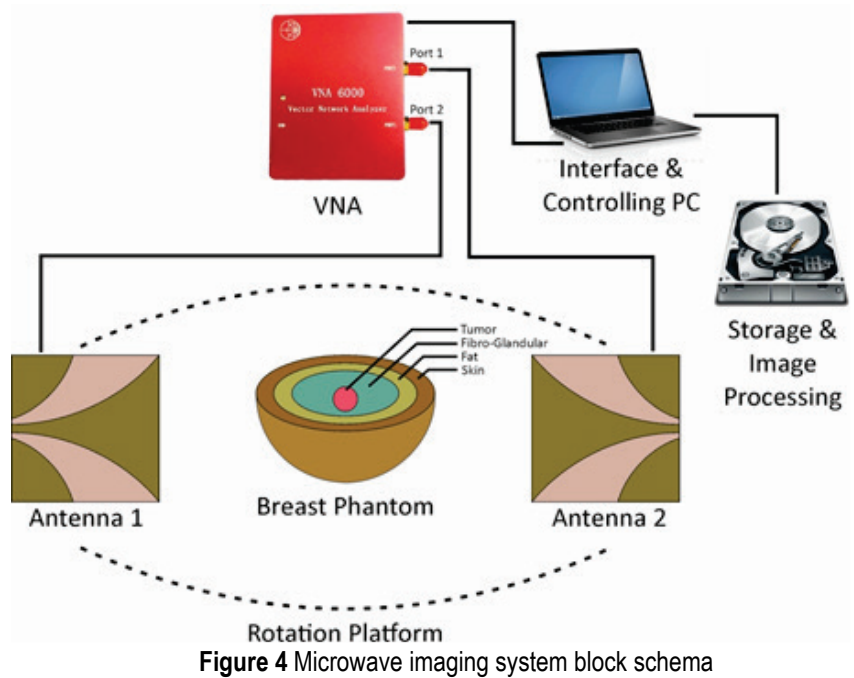

As shown in Fig. 5, a breast phantom of $12 \mathrm{~cm}$ in diameter was placed between two microstrip antennas, where one of them is a receiver, and the other is a transmitter. Tumors of different sizes were placed into the center, into the right of the center and into the left of the center of the fibro- glandular layer of the breast phantom, respectively. Experiments were performed by turning off two antennas mutually. The microwave imaging system and experimental setup were given in Fig. 4 and Fig. 5.

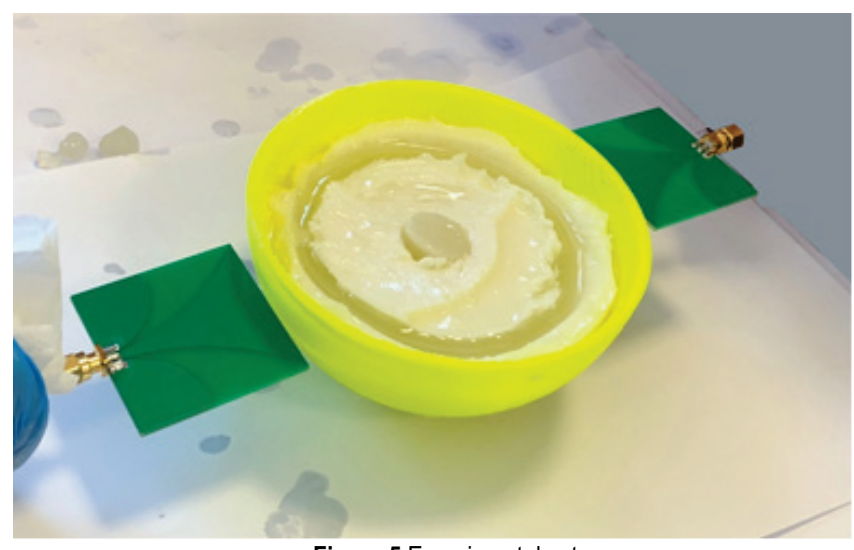

Figure 5 Experimental setup

The websites of major VNA producers such as Agilent and Rohde \& Schwarz [20] show that a wide range of VNA equipment is available. For the most advanced users, VNAs 
can provide high-precision signal measurements over a wide frequency range. This may confirm the use of GPRs and distinct areas, which often supply significant research results for a soil survey. The complexity of the equipment for more speculative research and field monitoring generally works at lower costs; some VNAs are now available as compact units suitable for field use.

Furthermore, many affordable portable PC-based VNAs are much cheaper on the market. The properties are given in Tab. 2. As a result, this device has the potential to be used in biomedical applications such as breast microwave imaging [20] and body bio-impedance measurement [21].

Table 2 The specifications of the miniVNA Tiny

\begin{tabular}{|l|l|}
\hline \multicolumn{1}{|c|}{ Parameter } & \multicolumn{1}{c|}{ Value } \\
\hline Frequency range & $1 \mathrm{MHz}$ to $3 \mathrm{GHz}$ \\
\hline Ports & 2 ports \\
\hline Output power & $-6 \mathrm{dBm}$ at $500 \mathrm{MHz}$ \\
\hline Dynamic range & Up to $70 \mathrm{~dB}$ at $500 \mathrm{MHz}$ \\
\hline Connectors & $2 \times \mathrm{SMA}$ \\
\hline Power consumption & $370 \mathrm{~mA}$ at $5 \mathrm{~V}$ (USB) \\
\hline Weight & $70 \mathrm{~g}$ \\
\hline Size & $66 \times 66 \times 28 \mathrm{~mm}$ \\
\hline Software & vna/J for Windows, Linux, and MacOS \\
\hline Impedance range $(Z)$ & 1 to 1000 ohm \\
\hline
\end{tabular}

In the experimental setup, we tested the performance of the antenna and the miniVNA to detect tumor cells in the breast. The frequency range of the miniVNA is between 100 $\mathrm{MHz}$ and $3 \mathrm{GHz}$, and the number of points is 636 . The miniVNA is connected to a PC via a USB port, and data are taken for further processing. The transmitted parameters bound up with the entire path between the two antennas where the tumor has passed twice during the entire rotation. The reflected signals often represent shallow depths below the skin layer because the signals projected from the other side of the phantom must move twice through the phantom and are significantly weakened. The reflected signals generally introduce shallow depths below the skin layer since signals reflected on the other side of the breast phantom must move twice from the phantom and are significantly weakened. Therefore, antennas with an exceptionally low loss of original return are ideal for detecting weakly reflected signals. The collected images were then processed, and the inner breast images were reconstructed. In this study, firstly the skin, fat and fibro-glandular layers were formed from the outer to the inner side in the form of a hemisphere. Then, tumor phantoms were formed in four different diameters and placed in three different locations in the fibro-glandular layer and measurements were taken. Firstly, the smallest tumor was placed into the center of the breast, then it was placed 2 $\mathrm{cm}$ from the right and $2 \mathrm{~cm}$ from the left of the center of the breast, respectively and measurements were taken with the help of the miniVNA. Afterwards, this step was repeated for other tumors as well. Furthermore, after the image was obtained in an experimental study without a tumor in the tissue center, a second experiment was created for the tissue containing a tumor in the center in order to compare the performance, and the same procedure was repeated. Next, inverse radon transform [22] was used to display the data obtained, and the results were successful. In the following figure (Fig. 6), the images show when there is no tumor (a), when the tumor is in the center of the breast (b), and when the tumor is $2 \mathrm{~cm}$ away from the center of the breast.

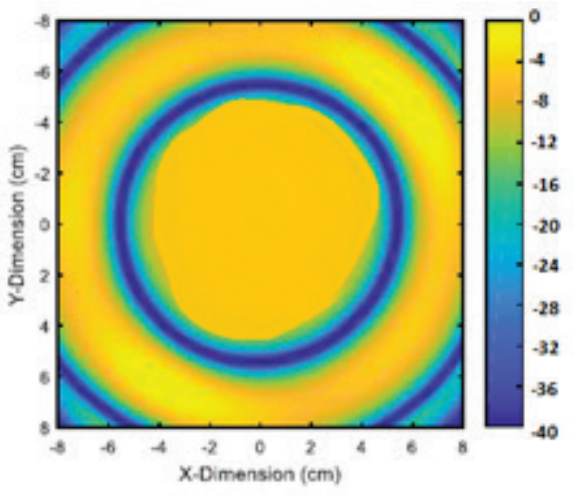

(a)

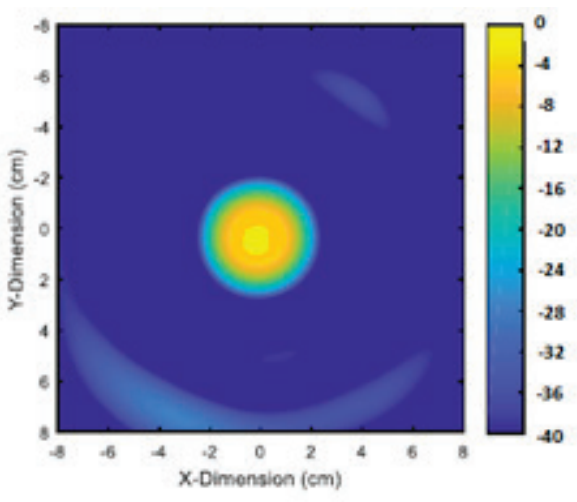

(b)

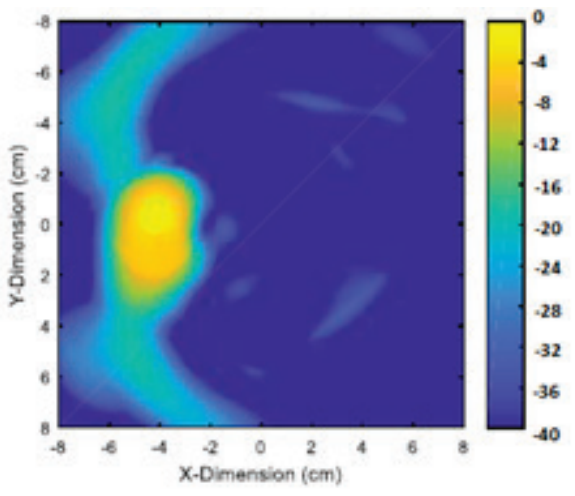

(c)

Figure 6 Images obtained by the inverse radon transform method (a) breast tissues without tumor, (b) when tumor is in the center of the breast (c) when tumor is $2 \mathrm{~cm}$ away from the center of the breast

\section{CONCLUSION}

In this study, the IRT (Inverse Radon Transform) method was used to reconstruct the appearance of a malignant tumor in the breast using the data obtained by experimental studies. Spherical tumors were obtained in four different diameters, and three different scenarios were established. The scenarios were as follows: measurements were taken by placing the first the smallest tumor at the center, then by placing it at a distance of $2 \mathrm{~cm}$ from the right of the center and then $2 \mathrm{~cm}$ from the left of the center. The same steps were followed for all tumors. Throughout the experiment, the antennas were mutually rotated with specific steps. The experimental phantom was illuminated by using a miniVNA in the frequency domain. The resolution of the image was made higher by increasing the sample points. The images were successfully obtained, and the IRT algorithm performed well in detecting breast cancer with microwaves. Although there are many vector network analyzers on the market, the miniVNA used in this study, which is portable and computer- 
based, has been shown to have potential in many biomedical applications such as breast cancer detection studies. Moreover, it can be seen that the IRT method is suitable for detecting breast cancer.

\section{Acknowledgment}

This research was partially supported by the Adana Alparslan Türkeş Science and Technology University Scientific Research Project Commission. Project Number: 18119003.

\section{REFERENCES}

[1] Olivotto, I. A., Bancej, C., Goel, V., Snider, J., McAuley, R. G., Irvine, B., Kan, L., Mirsky, D., Sabine, M. J., \& McGilly, R. (2001). Waiting times from abnormal breast screen to diagnosis in 7 Canadian provinces. Canadian Medical Association or its licensors (CMAJ), 165(3), 277-283.

[2] Fear, E. C., Li, X., Hagness, S. C., \& Stuchly, M. A. (2002). Confocal microwave imaging for breast cancer detection: Localization of tumors in three dimensions. IEEE Transactions on Biomedical Engineering, 49(8), 812-822. https://doi.org/10.1109/TBME.2002.800759

[3] Kurrant, D. J., Fear, E. C., \& Westwick, D. T. (2008). Tumor response estimation in radar-based microwave breast cancer detection. IEEE Transactions on Biomedical Engineering, 55(12), 2801-2811. https://doi.org/10.1109/TBME.2008.921164

[4] Servick, K. (2014). Breast cancer: a world of differences. American Association for the Advancement of Science. https://doi.org/10.1126/science.343.6178.1452

[5] Kahar, M., Ray, A., Sarkar, D., \& Sarkar, P. (2015). An UWB microstrip monopole antenna for breast tumor detection. Microwave and Optical Technology Letters, 57(1), 49-54. https://doi.org/10.1002/mop.28773

[6] Siegel, R. L., Miller, K. D., \& Jemal, A. (2019). Cancer statistics, 2019. CA: A Cancer Journal for Clinicians, 69(1), 734. https://doi.org/10.3322/caac.21551

[7] Jacobsen, K. K., Abraham, L., Buist, D. S., Hubbard, R. A., O’Meara, E. S., Sprague, B. L., Kerlikowske, K., Vejborg, I., Von Euler-Chelpin, M., \& Njor, S. H. (2015). Comparison of cumulative false-positive risk of screening mammography in the United States and Denmark. Cancer Epidemiology, 39(4), 656-663. https://doi.org/10.1016/j.canep.2015.05.004

[8] Nikolova, N. K. (2011). Microwave imaging for breast cancer. IEEE Microwave Magazine, 12(7), 78-94. https://doi.org/10.1109/MMM.2011.942702

[9] Irfan, N., Yagoub, M. C., \& Hettak, K. (2012). Design of a microstrip-line-fed inset patch antenna for RFID Applications. International Journal of Engineering and Technology, 4(5), 558. https://doi.org/10.7763/IJET.2012.V4.432

[10] Wang, L., Peng, H., \& Ma, J. (2017). Microwave breast imaging techniques and measurement systems. New Perspectives in Breast Imaging: IntechOpen. Ed. A. Malik. https://doi.org/10.5772/intechopen.69199

[11] Carr, K. L., Cevasco, P., Dunlea, P., \& Shaeffer, J. (2000). Radiometric sensing: An adjuvant to mammography to determine breast biopsy. IEEE MTT-S Int Microw Symp Dig. 2(2), 929-932. https://doi.org/10.1109/MWSYM.2000.863509

[12] Meaney, P. M., Fanning, M. W., Li, D., Poplack, S. P., \& Paulsen, K. D. (2000). A clinical prototype for active microwave imaging of the breast. IEEE Transactions on Microwave Theory and Techniques, 48(11), 1841-1853. https://doi.org/10.1109/22.883861
[13] Noghanian, S., Sabouni, A., \& Pistorius, S. (2006). A numerical approach to microwave imaging based on genetic algorithm optimization. Proc. SPIE 6177, Health Monitoring and Smart Nondestructive Evaluation of Structural and Biological Systems V, 61771B (15 March 2006). https://doi.org/10.1117/12.658572

[14] Fear, E. C., Meaney, P. M., \& Stuchly, M. A. (2003). Microwaves for breast cancer detection? IEEE Potentials, 22(1), 12-18. https://doi.org/10.1109/MP.2003.1180933

[15] Hancu, I., Roberts, J. C., Bulumulla, S., \& Lee, S.-K. (2015). On conductivity, permittivity, apparent diffusion coefficient, and their usefulness as cancer markers at MRI frequencies. Magnetic Resonance in Medicine, 73(5), 2025-2029. https://doi.org/10.1002/mrm.25309

[16] Porter, E., Fakhoury, J., Oprisor, R., Coates, M., \& Popović, M. (2010). Improved tissue phantoms for experimental validation of microwave breast cancer detection. Proceedings of the Fourth European Conference on Antennas and Propagation (EuCAP), 1-5.

[17] Lan, S.-W., Weng, M.-H., Yang, R.-Y., Chang, S.-J., Chung, Y.-S., Yu, T.-C., \& Wu, C.-S. (2016). Preparation of a carbon doped tissue-mimicking material with high dielectric properties for microwave imaging application. Materials, 9(7), 559. https://doi.org/10.3390/ma9070559

[18] Lazebnik, M., Madsen, E. L., Frank, G. R., \& Hagness, S. C. (2005). Tissue-mimicking phantom materials for narrowband and ultrawideband microwave applications. Physics in Medicine \& Biology, 50(18), 4245. https://doi.org/10.1088/0031-9155/50/18/001

[19] National Instruments. Introduction to Network Analyzer Measurements - Fundamentals and Background. http://download.ni.com/evaluation/rf/Introduction to Networ k_Analyzer_Measurements.pdf (Accessed: 30.04.2019)

[20] Byrne, D., Sarafianou, M., \& Craddock, I. J. (2017). Compound radar approach for breast imaging. IEEE Transactions on Biomedical Engineering, 64(1), 40-51. https://doi.org/10.1109/TBME.2016.2536703

[21] Patil, A. S. \& Ghongade, R. (2016). Design of bioimpedance spectrometer. International Conference on Advances in Computing, Communications and Informatics (ICACCI), 2724-2728. https://doi.org/10.1109/ICACCI.2016.7732473

[22] Boerner, W.-M., Ho, C.-M., \& Foo, B.-Y. (1981). Use of Radon's projection theory in electromagnetic inverse scattering. IEEE Transactions on Antennas and Propagation, 29(2), 336-341. https://doi.org/10.1109/TAP.1981.1142581

\author{
Authors' contacts: \\ Emine Avşar Aydin, Assistant Professor \\ (Corresponding author) \\ Adana Alparslan Türkeş Science and Technology University, \\ Department of Aeronautics Engineering \\ Adana/TURKEY \\ $+90-05318436781$ \\ eaydin@atu.edu.tr \\ rasvaenime@gmail.com
}

Selin Yabaci Karaoğlan, Assistant Professor

Adana Alparslan Türkeş Science and Technology University,

Department of Food Engineering

Adana/TURKEY

syabaci@atu.edu.tr 